\title{
Polymorphic Structures of One-Dimensional Ionic Crystals Confined in Carbon Nanotubes
}

\author{
R. Senga, Z. Liu, K. Hirose-Takai, K. Suenaga
}

Nanotube Research Center, National Institute of Advanced Industrial Science and Technology (AIST), Central 5, 1-1-1 Higashi, Tsukuba, 305-8565, Japan

Low-dimensional materials are attracting huge interest in many research fields as their confined size and reduced dimensionality allow the possibility of manipulation and utilization of their unusual properties. The interior of a carbon nanotube is one of the most ideal places for the synthesis of low-dimensional materials. Metallic [1], ionic [2], halogen [3], and dichalcogenide [4] crystals have been successfully filled in carbon nanotubes. In such nanocrystals, specific atoms, such as those at the edges or at the surface possessing a different coordination number, are more dominant than in bulk structures and can strongly affect the physical properties of the nanocrystalline materials. Therefore, a chemical analysis of a single atom and detailed structural examination are necessary for further understanding.

Herein, we describe the atomic structural analysis of ionic nanocrystals, encapsulated in single-walled carbon nanotubes (SWNTs), based on scanning transmission electron microscopy (STEM) and electron energy loss spectroscopy (EELS). We have demonstrated that the size and structure of the ionic crystals are clearly dependent on the size of the SWNTs.

In this study, we used SWNTs of various diameters [5] as templates and CsI crystals in different sizes as the material to be encapsulated. Cap-opened SWNTs were exposed to CsI gas. The resultant CsI crystals encapsulated in SWNTs were supported on W grids and observed with JEM-2100F equipped with a delta corrector and a cold-field emission gun operated at $60 \mathrm{kV}$, which could crucially reduce the damage of the samples caused by the electron beam. A fast Fourier transform (FFT) of a typical annular dark field (ADF) image shows that the microscope could resolve up to $0.108 \mathrm{~nm}$ in the STEM mode (not shown). The probe current was $\sim 40 \mathrm{pA}$.

Figure 1 details an example of a chemical analysis of a CsI nanocrystal encapsulated in a SWNT. The chemical maps (Fig. 1(c)-(e)) are constructed using the I $M_{4,5}$ edges and Cs $M_{4,5}$ edges in the EELS spectrum, as shown in Fig. 1(b). The possible structure, as shown in Fig. 1(f), based on the chemical maps and the high-resolution ADF image is body-centered cubic (bcc), the same as that of bulk materials. However, when the diameter of the SWNTs is reduced, the crystal forms $2 \times 2$ (Fig. 2 (a)) or $3 \times 3$ (Fig. 2(b)) structures derived from the face centered cubic (fcc; rocksalt) structure. We have confirmed that the transition from bcc to fcc structure occurs when the diameter of the SWNT is less than approximately $1.4 \mathrm{~nm}$. The properties of CsI crystals confined in nanotubes of much smaller diameters are also presented.

\section{References}

[1] L. Guan et al., Journal of the American Chemical Society. 130, 2162-2163 (2008).

[2] J. Sloan et al., Chemical Communications. 13, 1319-1332 (2002).

[3] L. Guan et al., Nano letters. 7, 1532-1535 (2007).

[4] Z. Wang et al., Journal of the American Chemical Society. 132, 13840-13847 (2010).

[5] Preparation of SWNT was supported by Dr Y. Sato and T. Saito. 
[6] This work was supported by the Japan Society for the Promotion of Science (JSAP) and JST Research Acceleration program.
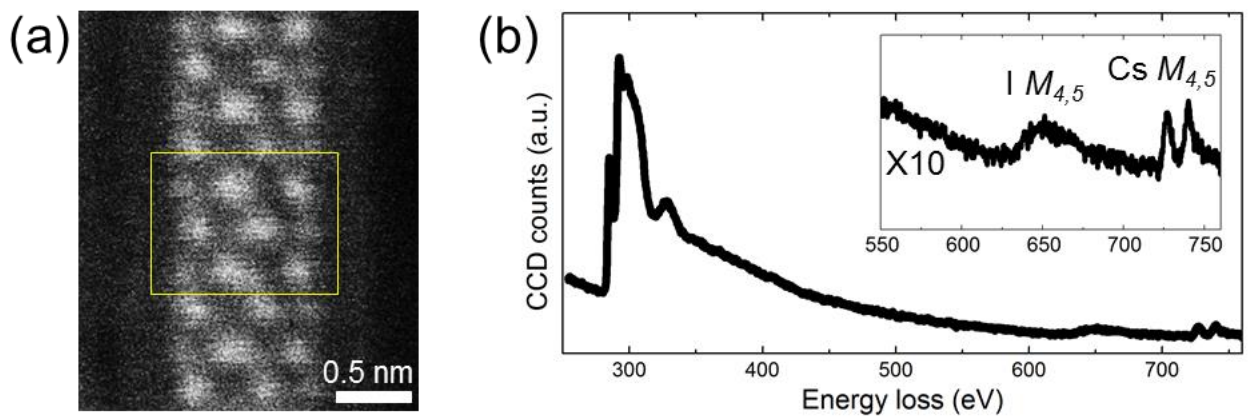

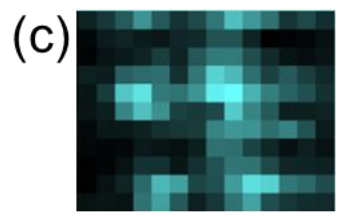

Cs

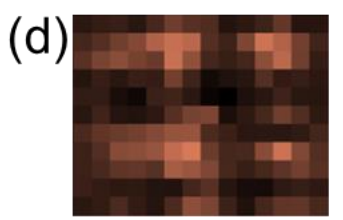

।

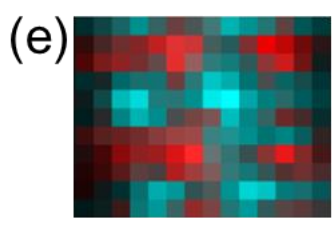

(f)

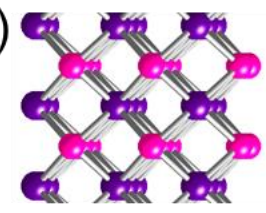

, e...

Figure 1. An example of chemical analysis of a CsI crystal encapsulated in a single walled carbon nanotube. (a) ADF image; (b) EELS spectrum of the region denoted in the square in (a); (c, d, e) EELS chemical maps for Cs (red), I (blue), and both Cs and I as CsI. (f) The corresponding atomic model for the chemical maps.

(a)

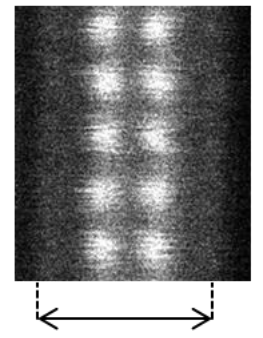

$1.1 \mathrm{~nm}$ (b)

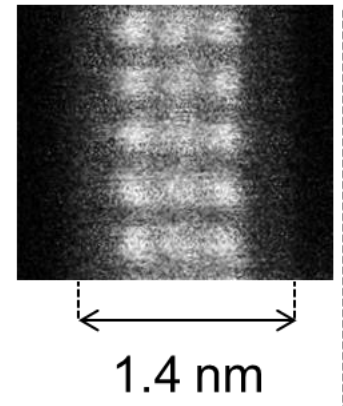

fcc structures (c)

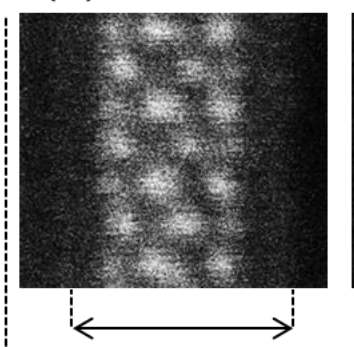

$1.5 \mathrm{~nm}$ (d)

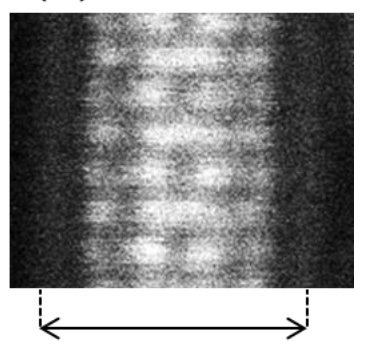

$1.7 \mathrm{~nm}$

\section{bcc structures}

Figure 2. Several structures of CsI nanocrystal encapsulated in SWNTs of different diameters. (a)-(d) ADF images of CsI nanocrystal encapsulated in SWNTs of diameters of 1.1, 1.4, 1.5 and $1.7 \mathrm{~nm}$, respectively. (a) and (b) indicate fcc structure. In contrast, (c) and (d) indicate bec structure. 